\title{
Tunneling in One-Dimensional non-Luttinger Electron Liquid
}

\author{
K. A. Matveev, B Dongxiao Yue, and L. I. Glazman \\ Theoretical Physics Institute, School of Physics and Astronomy, University of Minnesota, \\ 116 Church Str. SE, Minneapolis, MN 55455
}

\begin{abstract}
The conductance of a weakly interacting electron gas in the presense of a single scatterer is found at arbitrary strength of the scattering potential. At weak interaction, a simple renormalization group approach can be used instead of the standard bosonization procedure. Our technique allows to take into account the backscattering of electrons that leads to a non-Luttinger-liquid behavior of the low-temperature conductance. In the presence of magnetic field, the backscattering may give rise to a peak in differential conductance at bias equal to the Zeeman energy.
\end{abstract}

PACS numbers: 73.20.Dx, 73.40.Gk 
Recent progress in semiconductor technology renewed the interest in transport properties of one-dimensional (1D) electron systems. It is well known that in a clean short channel the electron transport is ballistic, and the conductance is quantized [1]. The ballistic conductance is destroyed, however, by scattering on impurities in longer channels [2]. It is reasonable to expect that in a sufficiently clean system it is possible to form a long $1 \mathrm{D}$ channel with a single impurity. Transport properties of such a system are determined by scattering of electrons on the impurity. In the simplest case of non-interacting electrons the conductance is related to the transmission coefficient by Landauer formula [3].

Electron-electron interaction in a 1D electron gas renormalizes significantly the scattering caused by an impurity potential [4]. The low-energy properties of a 1D interacting electron system are usually described by Luttinger model (see, e.g., [5]). This approach allows to treat the renormalizations of the impurity potential at any strength of interaction between electrons [6]. The resulting conductance $G$ demonstrates the power-law dependence on temperature at $T \rightarrow 0$. However, this method does not allow to calculate the conductance at higher temperatures where the power-law asymptotics fails. Besides, for the system of spin- $\frac{1}{2}$ electrons, the Luttinger model neglects backscattering in electron-electron collisions. The backscattering processes lead to additional renormalizations that affect the low-temperature conductance.

Below we present an alternative renormalization group ( $R G)$ approach that is valid only in the case of weakly interacting electrons. The advantage of our approach is in its ability to describe the conductance at any temperature. For a model with spinless electrons it allows us to show explicitly the crossover from the Fermi-gas to the low-temperature Luttinger liquid behavior. For the realistic case of spin- $\frac{1}{2}$ electrons, the backscattering processes can be incorporated into the renormalization procedure. Striking differences from Luttinger-liquid behavior occur in the case of a short-range interaction between electrons. The temperature dependence of conductance becomes non-monotonic, and application of a magnetic field creates a peak in the differential conductance at bias equal to the energy of the Zeeman splitting.

We start with a non-interacting 1D electron gas in the presense of a single scatterer. In the simplest case of a small-size scatterer we model its potential by $\delta$-function. The electron scattering is characterized by the transmission and reflection amplitudes $t_{0}$ and $r_{0}$. In terms of these amplitudes the scattering wave functions have the form:

$$
\begin{aligned}
& \psi_{k}(x)=\frac{1}{\sqrt{2}} \begin{cases}e^{i k x}+r_{0} e^{-i k x}, & x<0 \\
t_{0} e^{i k x}, & x>0\end{cases} \\
& \psi_{-k}(x)=\psi_{k}(-x) .
\end{aligned}
$$

Here $\psi_{k}$ and $\psi_{-k}$ describe the scattering of electrons incoming from the left and right correspondingly, $k>0$. Scattering disturbs the electron density around the barrier. In the presense of electron-electron interaction this modulation of density leads to an additional scattering of electrons. If the interaction potential $V(y-z)$ is weak, the correction to the wave functions (1) may be found in the Born approximation,

$$
\begin{aligned}
\delta \psi_{k}(x)= & \int_{-\infty}^{\infty} G_{k}(x, y) V_{H}(y) \psi_{k}(y) d y \\
& -\iint_{-\infty}^{\infty} G_{k}(x, y) V_{\mathrm{ex}}(y, z) \psi_{k}(z) d z d y .
\end{aligned}
$$


Here $G_{k}$ is the Green function for non-interacting electrons in the presense of the barrier. To solve the scattering problem we need only the asymptotics of the Green function at $x \rightarrow-\infty$. In this limit

$$
G_{k}(x, y)=\frac{1}{i \hbar v_{F}} \begin{cases}e^{i k(y-x)}+r_{0} e^{-i k(x+y)}, & y<0, \\ t_{0} e^{-i k(x-y)}, & y>0,\end{cases}
$$

where $v_{F}$ is the Fermi velocity. The correction (3) takes into account the electron scattering on both Hartree and exchange potentials:

$$
\begin{aligned}
V_{H}(y) & =\int_{-\infty}^{\infty} V(y-z) \delta n(z) d z \\
V_{\mathrm{ex}}(y, z) & =V(y-z) \int_{-k_{F}}^{k_{F}} \frac{d k}{2 \pi} \psi_{k}^{*}(y) \psi_{k}(z) .
\end{aligned}
$$

At large distances $|z| \gg k_{F}^{-1}$ the disturbance of density $\delta n(z)$ caused by the barrier decays as

$$
\delta n(z) \simeq \frac{\left|r_{0}\right|}{2 \pi|z|} \sin \left(2 k_{F}|z|-\arg r_{0}\right)
$$

The oscillations of density produce an oscillating Hartree potential commonly referred to as the Friedel oscillation, see Fig. 1. The asymptotics of the Hartree potential hints that in Born approximation the backscattering of a plain wave should diverge logarithmically at $k \rightarrow k_{F}$. Indeed, the explicit calculation based on Eqs. (3)-(6) gives the total first-order correction $\delta \mathcal{T}$ to the initial value $\mathcal{T}_{0}=\left|t_{0}\right|^{2}$ of the transmission coefficient

$$
\delta \mathcal{T}=-2 \alpha \mathcal{T}_{0}\left(1-\mathcal{T}_{0}\right) \ln \left(\frac{1}{\left|k-k_{F}\right| d}\right),
$$

where $d$ is the characteristic spatial scale of the interaction potential. The dimensionless parameter $\alpha$ characterizes the strength of interaction:

$$
\begin{gathered}
\alpha=\alpha_{2}-\alpha_{1}, \\
\alpha_{1}=\frac{V\left(2 k_{F}\right)}{2 \pi \hbar v_{F}}, \quad \alpha_{2}=\frac{V(0)}{2 \pi \hbar v_{F}} .
\end{gathered}
$$

Here $V(q)$ is the Fourier transformation of the interaction potential. The two terms proportional to $V(0)$ and $V\left(2 k_{F}\right)$ in Eq. (9) originate from the exchange and Hartree terms respectively. For the long-range interaction, $k_{F} d \gg 1$, the exchange term gives the leading contribution.

The lowest-order result (8) is applicable as long as $\alpha \ln \left(1 /\left|k-k_{F}\right| d\right) \ll 1$. At smaller $\left|k-k_{F}\right|$ we have to take into account the terms of higher orders in $\alpha$. Each term of the perturbation theory in $\alpha$ diverges at $k \rightarrow k_{F}$. The strongest divergence in the $n$-th term has the form $\alpha^{n} \ln ^{n}\left(1 /\left|k-k_{F}\right| d\right)$. We will find the transmission coefficient in the leading logarithm approximation that corresponds to summation of these most divergent terms. This task can be performed using a simple renormalization group approach, analogous to the "poor man's scaling" developed by Anderson [7] for the Kondo problem. 
The renormalization of the bare transmission coefficient $\mathcal{T}_{0}$ is caused by the interaction with the Fermi sea electrons. Since the maximum momentum transfer in a scattering event is determined by the spatial scale $d$ of the interaction, only electrons in the energy strip of halfwidth $D_{0}=\hbar v_{F} / d$ near the Fermi level contribute to the correction (8). First of all, we neglect the electron states with energies outside this strip. Then we transform our problem to a similiar one with a smaller bandwidth, $D<D_{0}$. The two problems are equivalent if we simultaneously renormalize $\mathcal{T}_{0}$ in order to take into account the interaction with the states excluded by this RG transformation. The renormalization of $\mathcal{T}_{0}$ found in Born approximation,

$$
\delta \mathcal{T}=-2 \alpha \mathcal{T}_{0}\left(1-\mathcal{T}_{0}\right) \ln \left(\frac{D_{0}}{D}\right),
$$

is similar to Eq. (8). The logarithmic factor corresonds to the integration over the strip of states excluded by this RG transformation.

We apply the RG transformation again, reducing the bandwidth $D$ step by step until it reaches the value $|\epsilon|$, where $\epsilon$ is the energy of the incoming electron counted from the Fermi level. Each step is accompanied by the renormalization (11) of the transmission coefficient. The sum of these small renormalizations may be found as a solution of the differential equation

$$
\frac{d \mathcal{T}}{d \ln \left(D_{0} / D\right)}=-2 \alpha \mathcal{T}(1-\mathcal{T}) .
$$

The initial condition at $D=D_{0}$ corresponds to the bare transmission coefficient $\mathcal{T}=\mathcal{T}_{0}$. The renormalized transmission coefficient for an electron with energy $\epsilon$ is given by the solution of the RG equation (12) at $D=|\epsilon|$,

$$
\mathcal{T}(\epsilon)=\frac{\mathcal{T}_{0}\left(|\epsilon| / D_{0}\right)^{2 \alpha}}{\mathcal{R}_{0}+\mathcal{T}_{0}\left(|\epsilon| / D_{0}\right)^{2 \alpha}},
$$

where $\mathcal{R}_{0}=\left|r_{0}\right|^{2}$ is the reflection coefficient. Formula (13) gives the power-law dependence $\mathcal{T}(\epsilon)$ at small $\epsilon$ that coinsides with the result of the Luttinger-liquid theory [6]. In contrast with this theory, our result (13) describes the behavior of transmission coefficient at all energies $|\epsilon|<D_{0}$. Besides, Eqs. (9) and (10) gives the microscopic definition of the exponent $\alpha$ in terms of the interaction potential. In the case of smooth potential Eq. (9) coinsides with the exponent found in [8].

Our approach allows also to find the temperature dependence of the linear conductance of a $1 \mathrm{D}$ interacting electron system with a single barrier. At $k_{B} T>D_{0}$ the conductance is given by the Landauer formula for an ideal Fermi gas, $G_{0}=\left(e^{2} / 2 \pi \hbar\right) \mathcal{T}_{0}$. At smaller temperatures the transmission coefficient is renormalized. The renormalization of transmission coefficient should be stopped at bandwidth $D \sim k_{B} T$ because of the smearing of the Fermi surface. As a result, the following temperature dependence of the linear conductance is found:

$$
G(T)=\frac{e^{2}}{2 \pi \hbar} \frac{\mathcal{T}_{0}\left(k_{B} T / D_{0}\right)^{2 \alpha}}{\mathcal{R}_{0}+\mathcal{T}_{0}\left(k_{B} T / D_{0}\right)^{2 \alpha}} .
$$

The differential conductance at a high voltage $e V>k_{B} T$ may be obtained by substitution $T \rightarrow\left(e / k_{B}\right) V$. 
Above we considered a model of spinless electrons. Experimentally this model can be realized by applying strong magnetic field that polarizes electron spins. For a system with spin degeneracy (zero magnetic field) the above theory should be revised. In particular, one has to take into account the backscattering processes involving two electrons with different spins. It is well-known [9] that the backscattering processes cause the renormalizations of interaction constants at low energies. These renormalizations are commonly studied in the framework of the following interaction Hamiltonian:

$$
\begin{aligned}
H_{\mathrm{int}}=\frac{1}{L} \sum_{k, p, q} \sum_{\sigma, \sigma^{\prime}} & g_{1} a_{k \sigma}^{\dagger} b_{p \sigma^{\prime}}^{\dagger} a_{p+2 k_{F}+q, \sigma^{\prime}} b_{k-2 k_{F}-q, \sigma}+g_{2} a_{k \sigma}^{\dagger} b_{p \sigma^{\prime}}^{\dagger} b_{p+q, \sigma^{\prime}} a_{k-q, \sigma} \\
& \left.+\frac{1}{2} g_{4}\left(a_{k \sigma}^{\dagger} a_{p \sigma^{\prime}}^{\dagger} a_{p+q, \sigma^{\prime}} a_{k-q, \sigma}+b_{k \sigma}^{\dagger} b_{p \sigma^{\prime}}^{\dagger} b_{p+q, \sigma^{\prime}} b_{k-q, \sigma}\right)\right] .
\end{aligned}
$$

Here $a_{k \sigma}^{\dagger}$ and $b_{k \sigma}^{\dagger}$ are the operators creating respectively left- and right-moving electrons with momentum $k$ and spin $\sigma$; interaction constant $g_{1}$ describes the backscattering, while $g_{2}$ and $g_{4}$ characterize the density-density interaction between the electrons moving in the opposite directions and in the same direction correspondingly. Unrenormalized constants $g_{1}, g_{2}$ and $g_{4}$ are determined by the Fourier components of the interaction potential,

$$
g_{1}=V\left(2 k_{F}\right), \quad g_{2}=g_{4}=V(0) .
$$

At low energies the constants $g_{1}$ and $g_{2}$ are changing [9] in the course of renormalization:

$$
\begin{aligned}
& g_{1}(D)=\frac{V\left(2 k_{F}\right)}{1+\frac{V\left(2 k_{F}\right)}{\pi \hbar v_{F}} \ln \frac{D_{0}}{D}}, \\
& g_{2}(D)=V(0)-\frac{1}{2} V\left(2 k_{F}\right)+\frac{1}{2} \frac{V\left(2 k_{F}\right)}{1+\frac{V\left(2 k_{F}\right)}{\pi \hbar v_{F}} \ln \frac{D_{0}}{D}} .
\end{aligned}
$$

Since $g_{4}$ remains unchanged, the equality $g_{2}=g_{4}$ is no longer valid. Therefore, we have to replace Eq. (12) with a new RG equation for the transmission coefficient $\mathcal{T}$ written in terms of constants $g_{1}, g_{2}$, and $g_{4}$ instead of $V(0)$ and $V\left(2 k_{F}\right)$. The first-order calculation with the Hamiltonian (15) gives

$$
\frac{d \mathcal{T}}{d \ln \left(D_{0} / D\right)}=-\frac{\left(g_{2}-2 g_{1}\right)}{\pi \hbar v_{F}} \mathcal{T}(1-\mathcal{T}) .
$$

Due to the spin degeneracy, the Hartree contribution proportional to $g_{1}$ contains extra factor of 2 [cf. Eq. (12) and (9)]. Taking into account the dependence (18) of $g_{1}$ and $g_{2}$ on scale $D$ and integrating Eq. (19), we find

$$
\mathcal{T}(\epsilon)=\frac{\mathcal{T}_{0}\left[1+2 \alpha_{1} \ln \frac{D_{0}}{\epsilon \mid}\right]^{3 / 2}\left|\frac{\epsilon}{D_{0}}\right|^{2 \alpha_{2}-\alpha_{1}}}{\mathcal{R}_{0}+\mathcal{T}_{0}\left[1+2 \alpha_{1} \ln \frac{D_{0}}{|\epsilon|}\right]^{3 / 2}\left|\frac{\epsilon}{D_{0}}\right|^{2 \alpha_{2}-\alpha_{1}}},
$$

where the parameters $\alpha_{1}$ and $\alpha_{2}$ are determined by Eq. (10). Similarly to Eq. (14), we can now find the conductance using the Landauer formula, 


$$
G(T)=\frac{e^{2}}{\pi \hbar} \frac{\mathcal{T}_{0}\left[1+2 \alpha_{1} \ln \frac{T_{0}}{T}\right]^{3 / 2}\left(\frac{T}{T_{0}}\right)^{2 \alpha_{2}-\alpha_{1}}}{\mathcal{R}_{0}+\mathcal{T}_{0}\left[1+2 \alpha_{1} \ln \frac{T_{0}}{T}\right]^{3 / 2}\left(\frac{T}{T_{0}}\right)^{2 \alpha_{2}-\alpha_{1}}}
$$

The above expression is the main result of this paper. In the absense of backscattering, $\alpha_{1}=0$, the existence of the spin degree of freedom leads only to a trivial factor of 2 , as compared to the conductance (14) in the spinless case. At $\alpha_{1}>0$ the backscattering gives rise to the logarithmic factors in Eq. (21) that determines the deviation from the power-law asymptotics of conductance at $T \rightarrow 0$. At sufficiently strong backscattering, $\alpha_{1}>\frac{1}{2} \alpha_{2}$, the temperature dependence of conductance changes qualitatively. As the temperature is lowered, the conductance first grows, reaching the maximum value at

$$
T \sim T_{0} \exp \left(-\frac{2 \alpha_{1}-\alpha_{2}}{\alpha_{1}\left(2 \alpha_{2}-\alpha_{1}\right)}\right),
$$

and then drops to zero at $T \rightarrow 0$.

The non-monotonic behavior of $G(T)$ is due to the renormalizations induced by backscattering of electrons with opposite spins. This part of the electron-electron interaction is responsible for the deviations from the Luttinger-liquid behavior at all energies. In the presence of magnetic field $B$ the backscattering is important only at energy scales exceeding $2 \mu_{B} B$, and the transmission coefficient $\mathcal{T}\left(|\epsilon| \geq 2 \mu_{B} B\right.$ ) is adequately described by Eq. (20). At energies below the Zeeman splitting $2 \mu_{B} B$ the backscattering is suppressed, and the Luttinger-liquid behavior restores. The renormalizations of the transmission coefficient for each spin direction follow the equation (12) for spinless electrons. The only difference occurs in the initial conditions that result from the renormalizations at higher energies according to $(20)$,

$$
\mathcal{T}_{0}^{*} \equiv\left(1-\mathcal{R}_{0}^{*}\right)=\left.\mathcal{T}\right|_{\epsilon=D_{0}^{*}}, \quad D_{0}^{*}=2 \mu_{B} B
$$

Thus the conductance at low temperatures $k_{B} T \ll \mu_{B} B$ is described by Eq. (14) with the substitution (23) and with the extra factor of 2 accounting for the two possible spin directions.

The electron subsystems with the opposite spins decouple at low energies because the Friedel oscillations produced by these subsystems have different periods if magnetic field is applied. The two periods correspond to the two different Fermi wave vectors, $k_{F \uparrow}$ and $k_{F \downarrow}$. At low temperatures only the electrons with energies close to the Fermi level contribute to the linear conductance. The low-energy electron is scattered effectively only by the same-spin Friedel oscillation, therefore the renormalizations occur in each spin subsystem independently. However, it is possible to make the electrons to scatter effectively on Friedel oscillations produced by the opposite-spin subsystem by applying a finite bias. This scattering shows up as a peak of the differential conductance at bias $V=2 \mu_{B} B / e$.

At a finite bias $V$ the current $I(V)$ can be expressed in terms of transmission coefficients $\mathcal{T}_{\sigma}(\epsilon, V)$ that in turn depends on voltage,

$$
I(V)=\frac{e}{2 \pi \hbar} \sum_{\sigma} \int_{-e V}^{0} \mathcal{T}_{\sigma}(\epsilon, V) d \epsilon .
$$


Here $\sigma= \pm 1$ characterizes the spin direction of tunneling electron, $\epsilon$ is its energy counted from the Fermi level at the left lead. We will calculate the transmission coefficient in the limit of strong barrier, $\mathcal{T}_{\sigma} \ll 1$. In the presence of bias voltage and Zeeman splitting, the RG equation (19) should be modified as follows:

$$
\begin{aligned}
\frac{d \ln \mathcal{T}_{\sigma}}{d \ln \left(D_{0} / D\right)}= & -\frac{g_{2}-g_{1}}{2 \pi \hbar v_{F}}[\theta(D-|\epsilon|)+\theta(D-|\epsilon+e V|)] \\
& +\frac{g_{1}}{2 \pi \hbar v_{F}}\left[\theta\left(D-\left|\epsilon-2 \sigma \mu_{B} B\right|\right)+\theta\left(D-\left|\epsilon+e V-2 \sigma \mu_{B} B\right|\right)\right] .
\end{aligned}
$$

We assumed here that the band of the half-width $D$ is centered at the energy $\epsilon$ of the tunneling electron. The RG transformation reducing the bandwidth leads to a logarithmic correction to $\mathcal{T}$ only as long as the Fermi momentum belongs to the new band. In our case, there are four relevant momenta corresponding to Friedel oscillations of electrons with the two spin directions in the two leads, and these constraints are represented by the four step-functions in the right-hand side of (25). The terms proportional to $g_{2}-g_{1}$ are due to the Friedel oscillations from the same-spin electrons which contribute to both exchange and Hartree potentials. The remaining terms are produced by interaction with the opposite-spin electrons.

The solution of RG equation (25) at $D \rightarrow 0$, found neglecting the renormalizations of the interaction constants [10], has the form:

$$
\begin{aligned}
\mathcal{T}_{\sigma}(\epsilon)= & \mathcal{T}_{0}\left|\frac{\epsilon}{D_{0}}\right|^{\alpha_{2}-\alpha_{1}}\left|\frac{\epsilon+e V-2 \sigma \mu_{B} B}{D_{0}}\right|^{-\alpha_{1}} \\
& \times\left|\frac{\epsilon+e V}{D_{0}}\right|^{\alpha_{2}-\alpha_{1}}\left|\frac{\epsilon-2 \sigma \mu_{B} B}{D_{0}}\right|^{-\alpha_{1}}
\end{aligned}
$$

This result combined with Eq. (24) allows to calculate the $I$ - $V$ characteristic. As it is clearly seen from Eq. (26), two singularities in $\mathcal{T}_{\sigma}(\epsilon)$ merge at $e V=2 \mu_{B} B$. As a result a singularity appears in the $I-V$ characteristic. The latter manifests itself as a peak in differential conductance,

$$
\delta G=\frac{e^{2}}{\pi \hbar} \mathcal{T}_{0}^{*} \frac{\alpha_{1}}{\alpha_{2}-2 \alpha_{1}}\left(1-\left|\frac{e V-2 \mu_{B} B}{2 \mu_{B} B}\right|^{\alpha_{2}-2 \alpha_{1}}\right),
$$

schematically shown in Fig. 2. The last result was obtained in the linear in $\mathcal{T}$ approximation that accounts for the processes in which electron crosses the tunnel barrier only once. This approximation is equivalent to the standard method of calculation of tunnel current in terms of the transmission coefficient and tunnel densities of states in the leads. Indeed, the expression (26) may be interpreted as the product of the bare transmission coefficient $\mathcal{T}_{0}$ and the power-law factors corresponding to the singular energy dependence of the tunnel densities of states. Apparently this method fails if the differential conductance becomes of the order of $e^{2} / \hbar$. This does not happen for a sufficiently long-range interaction potential, $g_{2}-2 g_{1}>0$. In the case of a short range potential, $g_{2}-2 g_{1}<0$, formula (27) is not applicable in a very narrow vicinity of the point $V=2 \mu_{B} B / e$.

In conclusion, we solved the problem of scattering on a single impurity for weakly interacting $1 \mathrm{D}$ electrons. This allowed us to find the system conductance at any temperature 
for spinless case (14) and for the real spin- $\frac{1}{2}$ electrons (21) as well. The electron-electron backscattering gives rise to deviations from the power-law temperature dependence of linear conductance at $T \rightarrow 0$. In the presence of a magnetic field, the backscattering creates a peak (27) in the differential conductance at bias $V=2 \mu_{B} B$.

The authors are grateful to H. U. Baranger, C. L. Kane, A. I. Larkin, and B. Z. Spivak for helpful discussions. This work was supported by NSF Grant DMR-9117341. 


\section{REFERENCES}

* On leave from the Institute of Solid State Physics, Chernogolovka, Moscow distr., 142432, Russia.

[1] C. W. J. Beenakker and H. van Houten, in Solid State Physics, Vol. 44, ed. by H. Ehrenreich and D. Turnbull (Academic Press, San Diego, 1991).

[2] G. Timp, in Semiconductors and Semimetals, ed. by M. A. Reed (Academic Press, New York, 1990).

[3] R. Landauer, IBM J. Res. Dev. 1, 223 (1957).

[4] D. C. Mattis, Phys. Rev. Lett. 32, 714 (1974).

[5] G. D. Mahan, Many-Particle Physics, (Plemun Press, New York, 1990), sec. 4.4.

[6] C. L. Kane and M. P. A. Fisher, Phys. Rev. Lett. 68, 1220 (1992).

[7] P. W. Anderson, J. Phys.C, 3, 2436 (1970).

[8] K. A. Matveev and L. I. Glazman, Phys. Rev. Lett. 70, 990 (1993).

[9] J. Solyom, Adv. Phys. 28, 201 (1979).

[10] This approximation is valid for small $g_{1}$, when $g_{1} \ln \left(D_{0} / \mu_{B} B\right) \ll 1$. If the latter condition is not satisfied, the result (27) should be corrected by the substitution of the renormalized values (17) and (18) of $g_{1,2}$ at $D=2 \mu_{B} B$ into the definition $\alpha_{1,2}=g_{1,2} / 2 \pi \hbar v_{F}$. 


\section{FIGURES}

FIG. 1. Total scattering potential $V(x)$. The central peak is the bare potential of the barrier. The wings represent the Friedel oscillation induced by the barrier.

FIG. 2. The differential conductance $G(V)=d I / d V$ of a quantum wire with a strong barrier in the presence of magnetic field $B$. The power-law behavior at $V \rightarrow 0$ is consistent with the Luttinger-liquid theory [6]. The power-law peak (27) at $V=2 \mu_{B} B / e$ is due to the scattering of electrons on the Friedel oscillation produced by the opposite-spin electrons. The curve was calculated for $\alpha_{1}=1 / 16, \alpha_{2}=3 / 8$. 